\title{
A Study on the Effect of MPE-FEC for 3D Video Broadcasting over DVB-H
}

\author{
Anil Aksay \\ Middle East Technical \\ University \\ Ankara, Turkey \\ anil@eee.metu.edu.tr \\ Antti Tikänmaki \\ Tampere University of \\ Technology \\ Tampere, Finland \\ antti.tikanmaki@tut.fi
}

\author{
M. Oguz Bici \\ Middle East Technical \\ University \\ Ankara, Turkey \\ mobici@eee.metu.edu.tr \\ Atanas Gotchev \\ Tampere University of \\ Technology \\ Tampere, Finland \\ atanas.gotchev@tut.fi
}

\author{
Döne Bugdayci \\ Middle East Technical \\ University \\ Ankara, Turkey \\ dbug@eee.metu.edu.tr \\ Gözde Bozdagi Akar \\ Middle East Technical \\ University \\ Ankara, Turkey \\ bozdagi@eee.metu.edu.tr
}

\begin{abstract}
Wireless networks are often error prone due to factors such as multi-path fading and interferences. In addition, the channel conditions of these networks are often non-stationary, such that the available bandwidth and channel error rates are changing over time with large variations. Therefore, they present a challenge for error-resilient video transmission. In order to maintain satisfactory QoS, a number of technologies have been proposed targeting different layers of the networks. DVB-H uses FEC for error protection and comes with an optional FEC tool (MPE-FEC) at the link-layer. In this paper, we propose to use the a priori knowledge of the transmitted media and apply MPE-FEC intelligently to provide better robustness by so-called Unequal Error Protection (UEP) mechanism. Extensive simulation results are given to show the effect of MPE-FEC on 3D compressed video under different channel conditions.
\end{abstract}

\section{Keywords}

DVB-H, stereo video, 3D, error resilient transmission

\section{INTRODUCTION}

Mobile TV has recently received a lot of attention worldwide with the advances in technologies such as Digital Multimedia Broadcasting (DMB), Digital Video Broadcasting Handheld (DVB-H) and MediaFLO [7]. On the other hand, $3 \mathrm{DTV}$ is a new approach to watching TV, shifting it from being a passive experience to an interactive and more realistic one. With the merge of these two technologies it will be possible to have 3DTV products based on cell phone platforms with switchable $2 \mathrm{D} / 3 \mathrm{D}$ autostereoscopic displays in the near future. Currently there are a number of projects

Permission to make digital or hard copies of all or part of this work for personal or classroom use is granted without fee provided that copies are not made or distributed for profit or commercial advantage and that copies bear this notice and the full citation on the first page. To copy otherwise, to republish, to post on servers or to redistribute to lists, requires prior specific permission and/or a fee.

Mobimedia '09, September 7-9, 2009, London, UK

Copyright 2009 ICST 978-963-9799-62-2/00/0004 ...\$5.00.

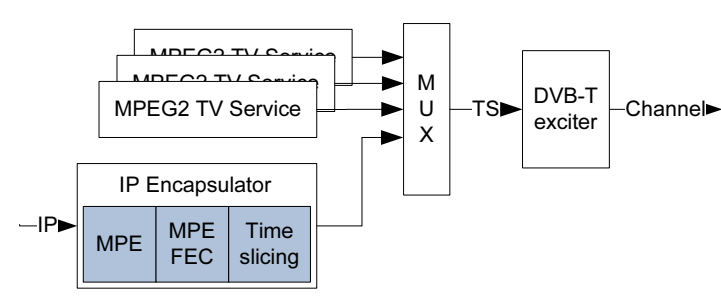

Figure 1: Basic elements of a DVB-H encapsulator and transmitter.

conducting research on this issue such as the Korean 3D TDMB [4], the European 3D Phone [8] and Mobile3DTV [14]. The latter one, specifically addresses the delivery of 3DTV to mobile users over DVB-H system.

There are several issues which have to be researched for 3D transmission over DVB-H such as the appropriate coding technique, error resilience, display factors etc. In this paper, we try to answer questions related to developing $3 \mathrm{D}$ video specific error resilient techniques and experimenting with delivery under different channel conditions. We propose to use the a priori knowledge of the transmitted media and apply MPE-FEC intelligently to provide better robustness by so-called Unequal Error Protection (UEP) mechanism.

The paper is structured as follows. Section 2 briefly summarizes the characteristics of DVB-H. Section 3 presents the experimental mobile 3DTV system used in this study. Section 4 outlines the coding approach used for mobile 3DTV. Section 5 addresses the simulated DVB-H channel. Section 6 analyzes the effect of error protection schemes on $3 \mathrm{D}$ video content. Section 7 describes the simulation environment and provides the experimental results together with a discussion. Finally Section 8 concludes the paper.

\section{DVB-H}

Basic elements of a DVB-H coder and transmitter are shown in Figure 1 [6] [2].

DVB-H is the extension of DVB Project for the mobile reception of digital terrestrial TV. It is based on the existing DVB-T physical layer with introduction of two new elements 
for mobility: MPE-FEC and time slicing. Time slicing enables the transmission of data in bursts rather than a continuous transmission; explicitly signaling the arrival time of the next burst in it so that the receiver can turn off in between and wake up before the next burst arrives. By this way the power consumption of the receiver is reduced.

Multi-Protocol Encapsulation is used for the carriage of IP datagram in MPEG2-TS. IP packets are encapsulated to MPE sections each of which consisting of a header, the IP datagram as a payload, and a 32-bit cyclic redundancy check (CRC) for the verification of payload integrity. On the level of the MPE, an additional stage of forward error correction (FEC) can also be added.This technique is called MPE-FEC and improves the $\mathrm{C} / \mathrm{N}$ and Doppler performance in mobile channels. To compute MPE-FEC, IP packets are filled into an $\mathrm{N}$ x 191 matrix where each square of the matrix has one byte of information and $\mathrm{N}$ denotes the number of rows in the matrix. The standard defines the value of $\mathrm{N}$ to be one of $256,512,768$ or 1024 . The datagram are filled into the matrix column-wise. Error correction codes (RS codes) are computed for each row and concatenated such that the final size of the matrix is of size Nx255. To adjust the effective MPE-FEC code rate, padding or puncturing can be used. Padding refers to filling the application data table partially with the data and the rest with zero whereas puncturing refers to discarding some of the rightmost columns of the RS-data table. The IP input streams coming from different sources are encapsulated and multiplexed for transmission according to the time slicing method. Figure 2 illustrates the MPE-FEC structure.

After encapsulation of IP packets and embedding into MPEG-2 Transport Stream (TS) packets, the next block is the DVB-T modulator. In addition to the $2 \mathrm{~K}$ and $8 \mathrm{~K}$ modes of DVB-T, DVB-H also uses an intermediate $4 \mathrm{~K}$ mode with a 4096-point Fast Fourier Transform (FFT) in the OFDM modulation. The objective of the $4 \mathrm{~K}$ mode is to improve the network planning flexibility. To further improve robustness of the DVB-T $2 \mathrm{~K}$ and $4 \mathrm{~K}$ modes in a mobile environment and impulse noise reception conditions, an in-depth symbol interleaver is also standardized.

\section{OVERVIEW OF 3D DVB-H SIMULATION SYSTEM}

The building blocks of the system used in this paper are illustrated in Figure 3 [3]. Stereo video content with right and left view is first compressed with a stereo video encoder (joint or simulcast). Resulting Network Abstraction Layer (NAL) units (NALU) are fed to the stereo video streamer. The streamer encapsulates the NAL units into Real Time Transport Protocol (RTP), User Datagram Protocol (UDP) and finally Internet Protocol (IP) datagram for each view separately. The resulting IP datagram are encapsulated in the DVB-H link layer where the Multi Protocol Encapsulation Forward Error Correction (MPE-FEC) and time slicing occurs. The left and right views are assigned different PIDs and encapsulated as different elementary streams. Therefore, left and right views are transmitted in different time slices or bursts. The link layer output MPEG-2 Transport Stream (TS) packets are passed to physical layer where the transmission signal is generated with a DVB-T modulator. After the transmission over a wireless channel, the receiver receives distorted signal and possibly erroneous TS pack-

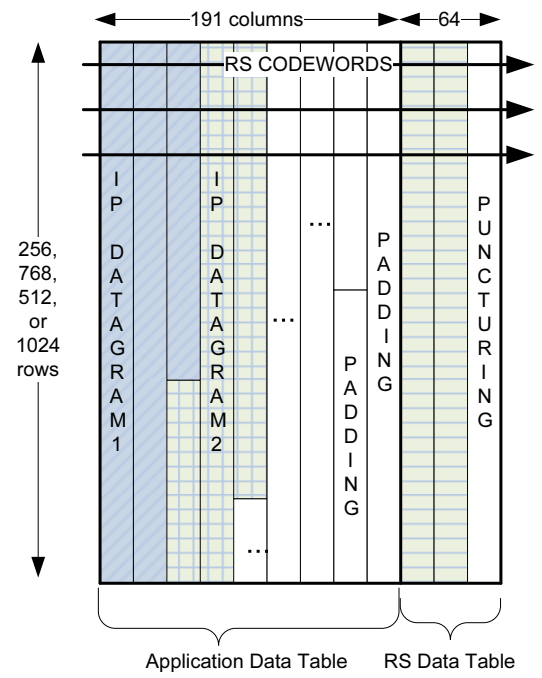

Figure 2: MPE-FEC frame structure.

ets are generated by the DVB-T modulator. The received stream is decoded using the section erasure method,i.e. the MPE-FEC frame is filled with contents of the error-free MPE and MPE-FEC sections and the empty bytes in the frame are marked as erasures, RS decoding is performed to reconstruct the lost data, and finally, the received and correctly reconstructed IP datagram are passed to the video client. IP datagram are handled in the stereo video streamer client and resulting NAL units are decoded with the stereo video decoder to generate right and left views. Finally, these views are combined with a special interleaving pattern to be displayed as 3D in the displayer.

\section{STEREO VIDEO CODER}

There are a few candidate formats for delivery and storage of 3D video: Simulcast coding, joint coding (Multi-View Coding, MVC), coding as Video-plus-depth (2D+depth) [1]. Hence, there are three main ways to encode these representations. In our system we use both simulcast and joint coding for the experimental study. In the future, these results are planned to be extended to other formats too.

MVC exploits temporal and inter-view redundancy by interleaving camera views and coding in a hierarchical manner. It is an amendment (Amendment 4) to H.264/AVC [12] and not yet completed. First draft is approved in October 2008 and second draft is approved in February 2009. MVC encoder used in our system is JMVM 3.0.2 [11]. Main prediction structure is quite complex introducing a lot of dependencies between images and views. This is valid even if the number of views to be coded is 2 (stereoscopic video). These dependencies make use of the redundancies present in both spatial and temporal directions to reduce the bit rate, however they also impose many restrictions in decoding and packet loss sensitivity. An alternative simplified structure is presented in [9], and shown to be very close to the main prediction structure in terms of overall coding efficiency. In this simplified prediction structure which is used in this study, the temporal prediction using hierarchical B-pictures 


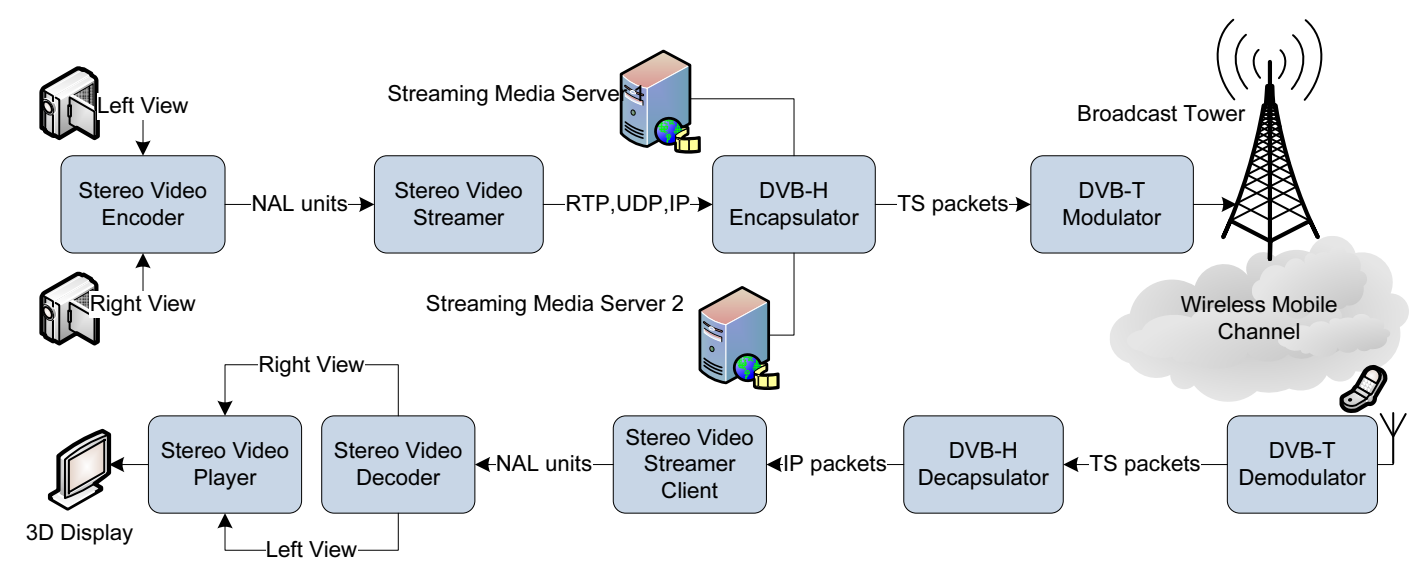

Figure 3: Block diagram of the experimental system.

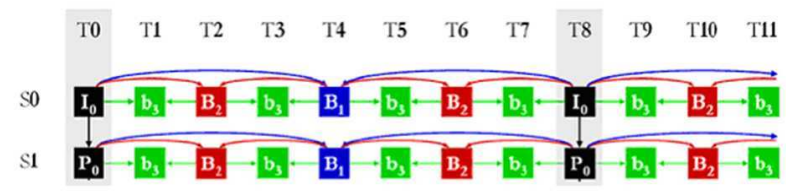

Figure 4: Simplified MVC coding scheme in case of two views.

remains unchanged when compared to original MVC prediction structure, but spatial references are only limited to anchor frames, such that spatial references are only allowed at the beginning of a group of pictures (GOP) between I and $\mathrm{P}$ pictures. This simplified version is shown in Figure 4 when the number of views $=2$.

\section{SIMULATED DVB-H CHANNEL}

For simulating the physical transmission channel, we have a MATLAB/Simulink tool [10] that models the DVB-T/H modulation and demodulation processes and the physical transmission channel. The channel is modeled as multipath Rayleigh fading channel with Additive White Gaussian Noise. In this paper a mobile use case with Cost 207 [5] radio channel model TU6, having maximum Doppler frequency of $24 \mathrm{~Hz}$ is used to obtain the channel specific error patterns. These patterns are then used for modeling the TS packet loss due to channel conditions. Since TU6 channel suffers more from dispersed errors caused by Doppler shift, the effect of MPE-FEC is more visible on this channel.

The simulations are carried out using the following DVB$\mathrm{H}$ transmission parameters: 16-QAM or QPSK, 1/2 convolutional code rate, $8 \mathrm{~K}$ OFDM mode, $1 / 4$ guard interval, $8 \mathrm{Mhz}$ bandwidth and 3 sec Delta-t. The channel was simulated using SNR levels ranging from $13 \mathrm{~dB}$ to $24 \mathrm{~dB}$ for $16-\mathrm{QAM}$ and from $9 \mathrm{~dB}$ to $18 \mathrm{~dB}$ for QPSK.

\section{EFFECT OF MPE-FEC RATE ASSIGN- MENT}

In [3], the necessity of using MPE-FEC for stereo video broadcasting over DVB-H was shown. In addition, significance of left and right views differ for simulcast and MVC coded videos. Unlike simulcast coding where left and right views have equal priorities due to independent coding; in case of MVC, left and right views possess different priorities. Since the right view is predicted from the left view, any error on the left view will directly affect the quality of the right view also causing a decrease on the overall quality.

The straightforward way to transmit joint or simulcast coded stereo video is to use equal error protection (same MPE-FEC rate) for both left and right views. However, this technique does not utilize the dependency of the views in case of MVC. It is crucial that right view can not be reconstructed regardless of how heavily it is protected or even received with no errors; if the left view is lost. Therefore use of Unequal Error Protection (UEP) between left and right views rather than Equal Error Protection (EEP) seems to be a better strategy for MVC.

We evaluated the impact of MPE-FEC by evaluating different MPE-FEC code rate assignments unequally on left and right sequences. In order to achieve different code rates, we employed puncture and padding operations.

\section{EXPERIMENTAL RESULTS}

The simulations presented in this paper are carried out using the stereo Horse sequence (480x272, 129 frames, 15 $\mathrm{Hz}, \mathrm{GOP}=16)$ prepared by KUK Filmproduktion $\mathrm{GmbH}$ and which is available in the stereo video database of Mobile3DTV Project [14]. The simulation process was repeated 80 times for each channel SNR condition in order to obtain figures corresponding to the average behavior of the channel. The quality of received and decoded video was measured by computing Y-PSNR and SSIM of the views using original, uncompressed video as the reference. In all the figures, PSNR values are in $\mathrm{dB}$ scale and calculated according to the following formulas where $D_{l}$ and $D_{r}$ represent the mean squared error in left and right views respectively. 


$$
\begin{gathered}
P S N R_{\text {joint }}=10 \cdot \log _{10}\left(\frac{255^{2}}{\left(D_{l}+D_{r}\right) / 2}\right) \\
P S N R_{\text {left(right) }}=10 \cdot \log _{10}\left(\frac{255^{2}}{D_{l}(r)}\right)
\end{gathered}
$$

SSIM indices are computed using the following formula [13].

$$
\operatorname{SSIM}(x, y)=\frac{\left(2 \mu_{x} \mu_{y}+c_{1}\right)\left(2 \operatorname{cov}_{x y}+c_{2}\right)}{\left(\mu_{x}^{2}+\mu_{y}^{2}+c_{1}\right)\left(\sigma_{x}^{2}+\sigma_{y}^{2}+c_{2}\right)}
$$

with $\mu_{x}$ the average of original image; $\mu_{y}$ the average of distorted image; $\sigma_{x}^{2}$ the variance of original image; $\sigma_{y}^{2}$ the variance of distorted image; $\operatorname{cov}_{x y}$ the covariance of distorted image; $c_{1}=6.5025, c_{2}=58.5225$.

Table 1 summarizes different MPE-FEC code rate assignment schemes used in the simulations. For each scheme, the resultant video PSNR and bitrate values after the compression are presented together with the final Transport Stream (TS) bitrate. For all cases, the video is encoded such that the resultant TS bitrate does not exceed the available bitrate defined by the transmission parameters presented in Section 5 with 16-QAM modulation. For example, if a scheme uses stronger FEC protection for a view, then it either reduces the video bitrate or assigns weaker FEC protection for the other view for a fair comparison. During the encoding, we adjust the video bitrate by varying $\mathrm{QP}$ values. In the table, EEP-1 and EEP-2 correspond to applying equal error protection to both streams, UEP-1 and UEP-2 correspond to applying different error protection rates to both streams and NO-PRO corresponds to streaming without error protection (MPE-FEC functionality switched off). We simulate simulcast coding with only EEP as the left and right sequences are coded independently.

Before we show the performance of UEP strategy, we first compare simulcast coding and MVC coding using the NOPRO and EEP-1 schemes shown in Table 1. The average PSNR plots for left view, right view and joint case are shown in Figures 5-7. As seen from the figures, the simulcast case is better only in a few very low channel SNR cases. This can be explained by the fact that in low channel SNR where MPE-FEC protection is not efficient, the frequent losses in left view also affects the right view for MVC case. However, as channel SNR increases, MVC case performs much better than simulcast case.

Next, we compare the different MPE-FEC rate allocation schemes shown in Table 1 for MVC coded video. The average PSNR figures for right view and joint case are shown in Figures 8-9. First of all, the results show a clear improvement of quality when the received MPE-FEC data is used especially in low channel SNR cases together with almost no loss performance for high channel SNR values. When FEC rate increases, again a clear improvement on quality is seen in low channel SNR cases.

Another observation from the Figures 8-9 is that, better quality can be achieved by UEP compared to EEP. This can be seen by comparing EEP-2 and UEP-2. In EEP-2 scheme, both views use a FEC rate of $7 / 8$. In UEP-2 scheme, right view is not protected at all and left view is protected with a FEC rate of $3 / 4$, which is stronger than the $7 / 8$ of EEP-2. Therefore, UEP-2 case is almost equivalent to

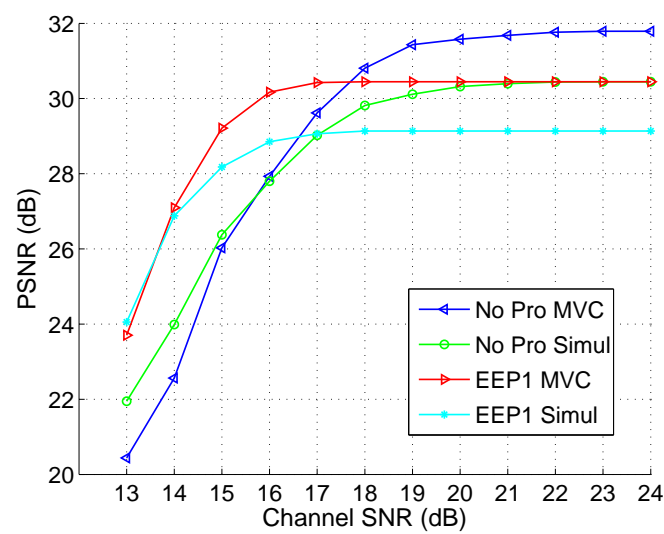

Figure 5: PSNR comparison of MVC and simulcast coding strategies for left sequence. Modulation: 16QAM

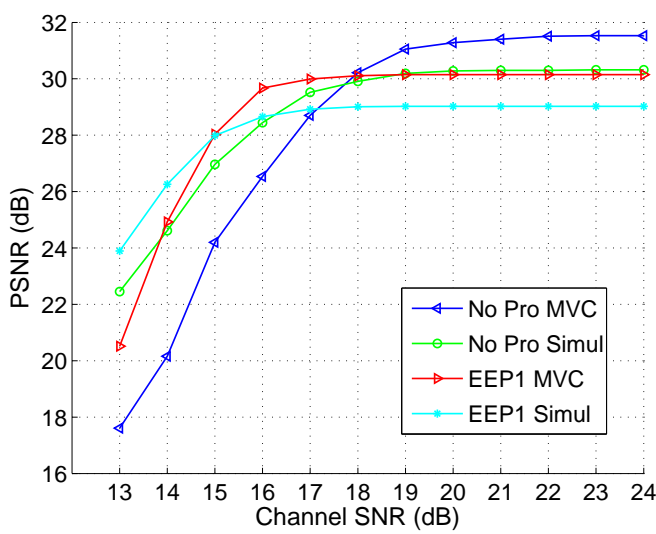

Figure 6: PSNR comparison of MVC and simulcast coding strategies for right sequence. Modulation: 16-QAM

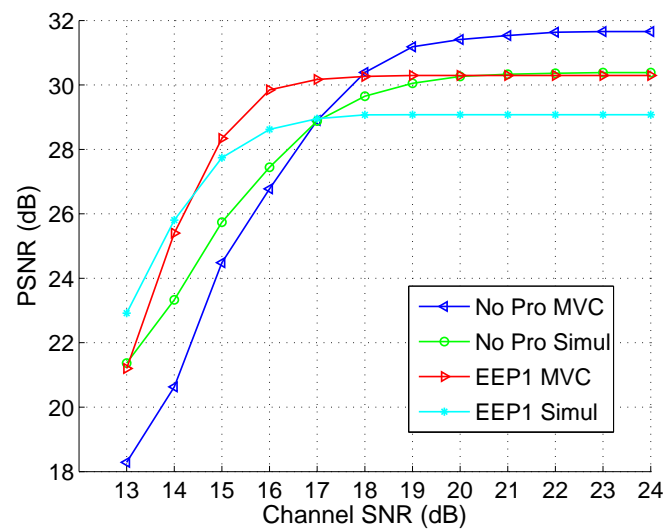

Figure 7: Joint PSNR comparison of MVC and simulcast coding strategies for both sequences. Modulation: 16-QAM 
Table 1: Video coding and MPE-FEC rate values used in the simulations with 16-QAM transmission

\begin{tabular}{|c|cc|ccc|ccc|ccc|}
\hline MVC & \multicolumn{3}{|c|}{ FEC Rate } & \multicolumn{3}{c|}{ PSNR } & \multicolumn{2}{|c|}{ Video bit rate (Kbps) } & \multicolumn{3}{c|}{ TS bit rate (Kbps) } \\
& $\mathrm{L}$ & $\mathrm{R}$ & $\mathrm{L}$ & $\mathrm{R}$ & Joint & $\mathrm{L}$ & $\mathrm{R}$ & Total & $\mathrm{L}$ & $\mathrm{R}$ & Total \\
\hline NO-PRO & NO FEC & NO FEC & 31.79 & 31.53 & 31.66 & 193.25 & 122.95 & 316.20 & 236.62 & 153.90 & 390.52 \\
EEP-1 & $3 / 4$ & $3 / 4$ & 30.45 & 30.15 & 30.30 & 143.44 & 87.62 & 231.06 & 235.39 & 159.67 & 395.06 \\
EEP-2 & $7 / 8$ & $7 / 8$ & 31.00 & 30.72 & 30.86 & 163.08 & 101.51 & 264.59 & 229.62 & 151.80 & 381.42 \\
UEP-1 & $3 / 4$ & $7 / 8$ & 30.45 & 30.64 & 30.54 & 143.44 & 106.96 & 250.40 & 235.39 & 159.49 & 394.89 \\
UEP-2 & $3 / 4$ & NO FEC & 30.45 & 31.38 & 30.89 & 143.44 & 139.01 & 282.45 & 235.39 & 173.66 & 409.05 \\
\hline Simulcast & $\mathrm{L}$ & $\mathrm{R}$ & $\mathrm{L}$ & $\mathrm{R}$ & Joint & $\mathrm{L}$ & $\mathrm{R}$ & Total & $\mathrm{L}$ & $\mathrm{R}$ & Total \\
\hline NO-PRO & NO FEC & NO FEC & 30.45 & 30.32 & 30.38 & 143.44 & 143.43 & 286.87 & 179.78 & 179.61 & 359.39 \\
EEP-1 & $3 / 4$ & $3 / 4$ & 29.14 & 29.02 & 29.08 & 104.68 & 103.27 & 207.95 & 176.11 & 174.53 & 350.64 \\
EEP-2 & $7 / 8$ & $7 / 8$ & 29.79 & 29.67 & 29.73 & 123.51 & 122.43 & 245.94 & 178.91 & 176.63 & 355.54 \\
\hline
\end{tabular}

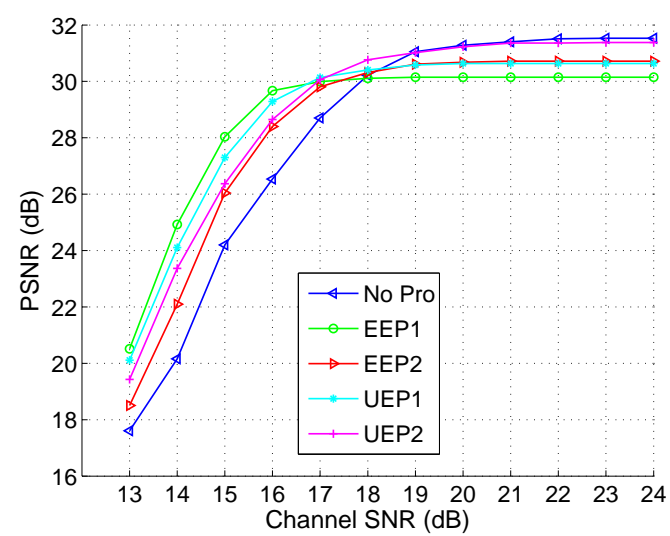

Figure 8: PSNR comparison of MPE-FEC rate assignment strategies for right sequence. Coding: MVC, Modulation: 16-QAM

using parity bits of the right view in EEP-2 for stronger protection of the left view. The figures show that, UEP2 achieves significant PSNR gains especially in low channel SNR cases. The comparison of EEP-1 and UEP-1 is different from EEP-2 and UEP-2 comparison. The reason is that both EEP-1 and UEP-1 use same FEC protection rate for left view but for right view, UEP-1 method uses the excessive bitrate earned from protecting right view weaker to encode video with a better quality. As a result, EEP-1 performs better in low channel SNR as it employs stronger protection and performs worse in good channel conditions as UEP-1 encodes video with a better quality.

Apart from 16-QAM modulation, we also repeated the same experiments with QPSK modulation. Since QPSK results in half bit rate compared to 16-QAM, we adjust all the video bitrate parameters in Table 1 such that the video bitrates are halved as well. Corresponding parameters are tabulated in Table 2. We show the results of simulcast-mvc coding comparison and different MPE-FEC rate schemes for average joint PSNR case in Figures 10 and 11. The results for QPSK modulation are in agreement with 16-QAM case, confirming our deductions. Comparing the results of QPSK and 16-QAM modulation, QPSK achieves higher PSNR values in very low channel SNR values. This is expected since QPSK is more robust to channel errors than 16-QAM. However, since the data bitrate is halved, maximum quality that

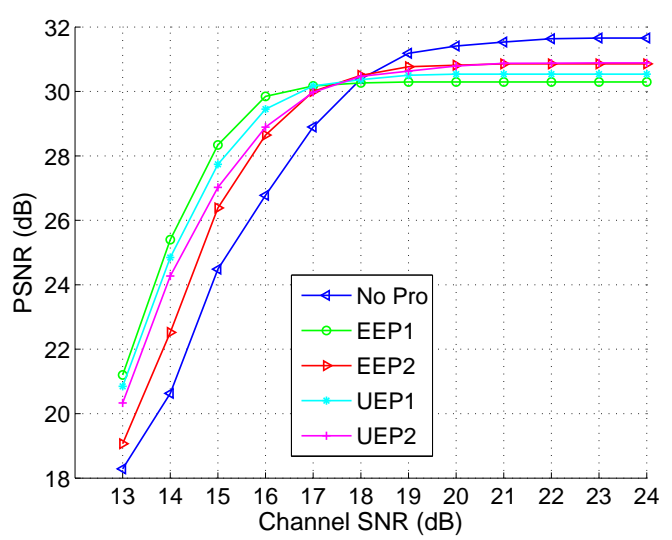

Figure 9: Joint PSNR comparison of MPE-FEC rate assignment strategies for both sequences. Coding: MVC, Modulation: 16-QAM

can be achieved by QPSK is much lower than 16-QAM and as the channel conditions get better, 16-QAM performs significantly better.

Finally, we tabulate the results of all the aforementioned experiments in Tables 3-4. The average distortions in Table 3 are given for PSNR values and the ones in Table 4 are given in SSIM metric.

\section{CONCLUSIONS AND FUTURE WORK}

In this paper, we study the impact of MPE-FEC over the quality of the delivered stereo video for different channel conditions. As shown in the experimental results, although MPE-FEC provides the much needed data robustness for $3 \mathrm{D}$ video transmission in wireless channels, under very erroneous conditions it may fail. In order to overcome this we use a priori knowledge of the media to differentially protect data using FEC. High priority (left) video is well protected and low priority (right) video is less protected. The results show that the protection strategy to be used highly depends on the channel conditions. In our future studies we will utilize different priority assignments for better UEP.

\section{ACKNOWLEDGMENTS}

MOBILE3DTV project has received funding from the European Community's ICT programme in the context of the 
Table 2: Video coding and MPE-FEC rate values used in the simulations with QPSK transmission

\begin{tabular}{|c|cc|ccc|ccc|ccc|}
\hline MVC & FEC Rate & \multicolumn{4}{|c|}{ PSNR } & \multicolumn{3}{|c|}{ Video bit rate (Kbps) } & \multicolumn{3}{c|}{ TS bit rate (Kbps) } \\
& L & R & L & R & Joint & L & R & Total & L & R & Total \\
\hline NO-PRO & NO FEC & NO FEC & 28.56 & 28.11 & 28.34 & 90.08 & 50.85 & 140.93 & 119.62 & 72.75 & 192.37 \\
EEP-1 & $3 / 4$ & $3 / 4$ & 27.45 & 26.94 & 27.19 & 79.05 & 42.91 & 121.96 & 120.32 & 73.28 & 193.60 \\
EEP-2 & $7 / 8$ & $7 / 8$ & 28.09 & 27.57 & 27.83 & 65.37 & 35.96 & 101.33 & 115.95 & 74.15 & 190.10 \\
UEP-1 & $3 / 4$ & $7 / 8$ & 27.45 & 27.39 & 27.42 & 65.37 & 45.88 & 111.26 & 115.95 & 78.17 & 194.12 \\
UEP-2 & $3 / 4$ & NO FEC & 27.45 & 27.86 & 27.65 & 65.37 & 57.08 & 122.45 & 115.95 & 79.92 & 195.87 \\
\hline Simulcast & $\mathrm{L}$ & $\mathrm{R}$ & $\mathrm{L}$ & $\mathrm{R}$ & Joint & $\mathrm{L}$ & $\mathrm{R}$ & Total & $\mathrm{L}$ & $\mathrm{R}$ & Total \\
\hline NO-PRO & NO FEC & NO FEC & 27.45 & 27.35 & 27.40 & 65.37 & 64.04 & 129.41 & 90.24 & 88.49 & 178.73 \\
EEP-1 & $3 / 4$ & $3 / 4$ & 26.53 & 26.47 & 26.50 & 48.67 & 47.11 & 95.78 & 90.24 & 88.49 & 178.73 \\
EEP-2 & $7 / 8$ & $7 / 8$ & 27.01 & 26.94 & 26.97 & 57.63 & 56.26 & 113.89 & 91.29 & 89.37 & 180.66 \\
\hline
\end{tabular}

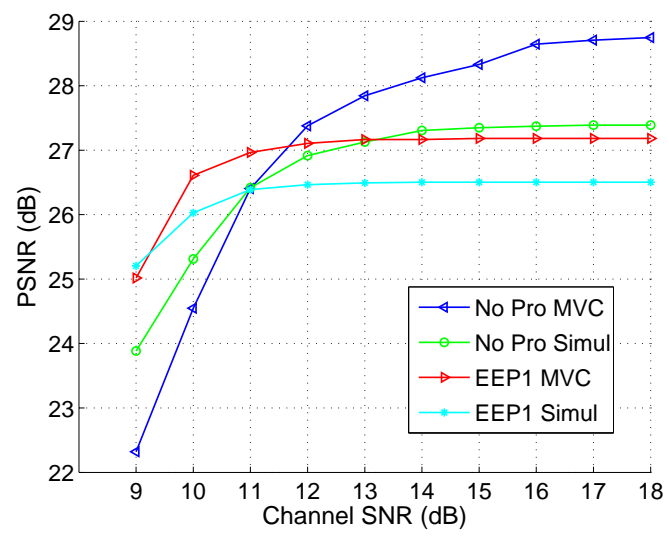

Figure 10: Joint PSNR comparison of MVC and simulcast coding strategies for both sequences. Modulation: QPSK

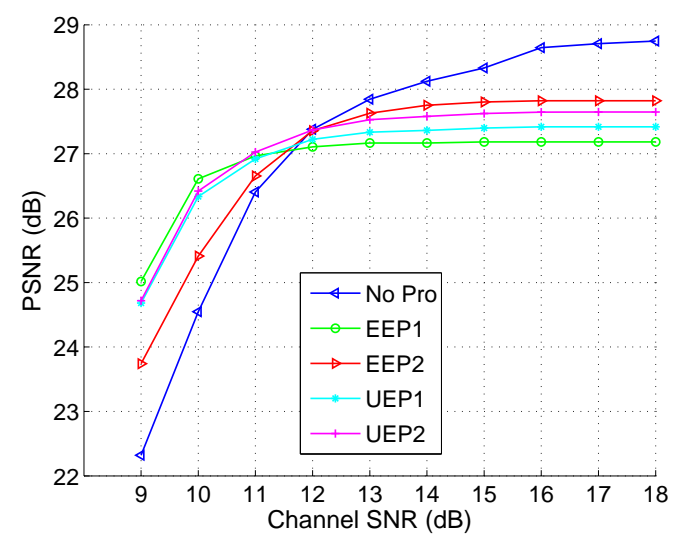

Figure 11: Joint PSNR comparison of MPE-FEC rate assignment strategies for both sequences. Coding: MVC, Modulation: QPSK
Seventh Framework Programme (FP7/2007-2011) under grant agreement nř 216503. The text reflects only the authors views and the European Community or other project partners are not liable for any use that may be made of the information contained herein. A. Aksay and M. Oguz Bici are partially supported by TUBITAK.

\section{REFERENCES}

[1] ISO/IEC JTC1/SC29/WG11 Overview of 3D Video Coding, May 2008. Doc. N9784.

[2] ETSI, Digital Video Broadcasting (DVB): DVB-H implementation guidelines, 2009. TR 102377 V1.3.1.

[3] M. O. Bici, A. Aksay, A. Tikanmaki, A. Gotchev, and G. Bozdagi Akar. Stereo Video Broadcasting Simulation for DVB-H. In NEM-Summit'08, 2008.

[4] S. Cho, N. Hur, J. Kim, K. Yun, and S. Lee. Carriage of 3D audio-visual services by T-DMB. Electronics and Telecommunications Research Institute, Republic of Korea, in Proc ICME, 2006.

[5] E. Failli. Digital land mobile radio. Final report of COST 207, 1989

[6] G. Faria, J. Henriksson, E. Stare, and P. Talmola. DVB-H: Digital broadcast services to handheld devices. Proceedings of the IEEE, 94(1):194-209, 2006.

[7] B. Furht and S. Ahson. Handbook of Mobile Broadcasting: $D V B-H, D M B, I S D B-T$, and MediaFLO. Auerbach Publications, 2008.

[8] http://www.3dphone.org/. 3dphone project.

[9] P. Merkle, A. Smolic, K. Mueller, and T. Wiegand. Comparative study of mvc prediction structures. Joint Video Team of ISO/IEC MPEG \& ITU-T VCEG.

[10] M. Oksanen, A. Tikanmaki, A. Gotchev, and I. Defee. Delivery of 3D Video over DVB-H: Building the Channel. In NEM-Summit'08, 2008.

[11] P. Pandit, A. Vetro, and Y. Chen. Jmvm 3 software. ITU-T JVTV208, 2007.

[12] A. Vetro, P. Pandit, H. Kimata, and A. Smolic. Joint draft 8.0 on multiview video coding. Joint Video Team (JVT) of ISO/IEC MPEG ITU-T VCEG ISO/IEC JTC1/SC29/WG11 and ITU-T SG16 Q.6, 2007.

[13] Z. Wang, A. Bovik, H. Sheikh, and E. Simoncelli. Image quality assessment: From error measurement to structural similarity. IEEE transactions on image processing, 13(4):600-612, 2004.

[14] www.mobile3dtv.eu. Mobile3dtv: Mobile 3dtv content delivery over dvb-h system. 
Table 3: Simulation results in average PSNR for 16-QAM and QPSK modulations

\begin{tabular}{|c|ccccc|ccc|}
\hline Channel SNR & \multicolumn{5}{|c|}{ MVC 16QAM } & \multicolumn{3}{c|}{ Simulcast 16QAM } \\
& No Pro & EEP1 & EEP2 & UEP1 & UEP2 & No Pro & EEP1 & EEP2 \\
\hline 13 & 18.287 & 21.202 & 19.069 & 20.849 & 20.331 & 21.369 & 22.917 & 21.635 \\
14 & 20.632 & 25.402 & 22.523 & 24.848 & 24.273 & 23.332 & 25.805 & 24.037 \\
15 & 24.483 & 28.341 & 26.385 & 27.739 & 27.024 & 25.739 & 27.743 & 26.585 \\
16 & 26.777 & 29.847 & 28.644 & 29.452 & 28.892 & 27.445 & 28.622 & 28.115 \\
17 & 28.898 & 30.170 & 30.000 & 30.171 & 29.973 & 28.879 & 28.953 & 29.031 \\
18 & 30.387 & 30.265 & 30.499 & 30.368 & 30.467 & 29.651 & 29.070 & 29.599 \\
19 & 31.186 & 30.295 & 30.766 & 30.501 & 30.631 & 30.052 & 29.078 & 29.694 \\
20 & 31.408 & 30.295 & 30.815 & 30.539 & 30.787 & 30.265 & 29.078 & 29.718 \\
21 & 31.533 & 30.295 & 30.855 & 30.539 & 30.874 & 30.332 & 29.078 & 29.727 \\
22 & 31.634 & 30.295 & 30.855 & 30.539 & 30.876 & 30.364 & 29.078 & 29.727 \\
23 & 31.657 & 30.295 & 30.855 & 30.539 & 30.888 & 30.382 & 29.078 & 29.727 \\
24 & 31.657 & 30.295 & 30.855 & 30.539 & 30.888 & 30.382 & 29.078 & 29.727 \\
\hline Channel SNR & \multicolumn{9}{|c}{ MVC QPSK } & & Simulcast QPSK \\
& No Pro & EEP1 & EEP2 & UEP1 & UEP2 & No Pro & EEP1 & EEP2 \\
\hline 9 & 22.320 & 25.017 & 23.742 & 24.683 & 24.718 & 23.884 & 25.203 & 24.442 \\
10 & 24.549 & 26.610 & 25.410 & 26.335 & 26.420 & 25.313 & 26.028 & 25.479 \\
11 & 26.405 & 26.966 & 26.655 & 26.919 & 27.025 & 26.418 & 26.389 & 26.329 \\
12 & 27.379 & 27.105 & 27.357 & 27.223 & 27.368 & 26.917 & 26.465 & 26.716 \\
13 & 27.844 & 27.165 & 27.628 & 27.333 & 27.527 & 27.128 & 26.492 & 26.876 \\
14 & 28.124 & 27.165 & 27.752 & 27.361 & 27.578 & 27.305 & 26.502 & 26.924 \\
15 & 28.330 & 27.182 & 27.803 & 27.398 & 27.623 & 27.349 & 26.502 & 26.969 \\
16 & 28.646 & 27.182 & 27.822 & 27.416 & 27.644 & 27.372 & 26.502 & 26.974 \\
17 & 28.707 & 27.182 & 27.822 & 27.416 & 27.646 & 27.388 & 26.502 & 26.974 \\
18 & 28.748 & 27.182 & 27.822 & 27.416 & 27.647 & 27.388 & 26.502 & 26.974 \\
\hline
\end{tabular}

Table 4: Simulation results in average SSIM for 16-QAM and QPSK modulations

\begin{tabular}{|c|ccccc|ccc|}
\hline Channel SNR & \multicolumn{5}{|c|}{ MVC 16QAM } & \multicolumn{3}{c|}{ Simulcast 16QAM } \\
& No Pro & EEP1 & EEP2 & UEP1 & UEP2 & No Pro & EEP1 & EEP2 \\
\hline 13 & 0.371 & 0.495 & 0.393 & 0.471 & 0.457 & 0.464 & 0.544 & 0.481 \\
14 & 0.474 & 0.670 & 0.559 & 0.656 & 0.637 & 0.578 & 0.677 & 0.605 \\
15 & 0.632 & 0.786 & 0.711 & 0.768 & 0.749 & 0.692 & 0.751 & 0.716 \\
16 & 0.723 & 0.840 & 0.798 & 0.830 & 0.813 & 0.757 & 0.783 & 0.774 \\
17 & 0.801 & 0.853 & 0.846 & 0.853 & 0.848 & 0.807 & 0.794 & 0.805 \\
18 & 0.854 & 0.856 & 0.864 & 0.859 & 0.862 & 0.833 & 0.798 & 0.824 \\
19 & 0.883 & 0.857 & 0.872 & 0.863 & 0.867 & 0.845 & 0.798 & 0.827 \\
20 & 0.889 & 0.857 & 0.874 & 0.864 & 0.871 & 0.851 & 0.798 & 0.828 \\
21 & 0.894 & 0.857 & 0.875 & 0.864 & 0.874 & 0.854 & 0.798 & 0.828 \\
22 & 0.897 & 0.857 & 0.875 & 0.864 & 0.874 & 0.854 & 0.798 & 0.828 \\
23 & 0.898 & 0.857 & 0.875 & 0.864 & 0.874 & 0.855 & 0.798 & 0.828 \\
24 & 0.898 & 0.857 & 0.875 & 0.864 & 0.874 & 0.855 & 0.798 & 0.828 \\
\hline Channel SNR & \multicolumn{9}{|c}{ MVC QPSK } & & & Simulcast QPSK \\
& No Pro & EEP1 & EEP2 & UEP1 & UEP2 & No Pro & EEP1 & EEP2 \\
\hline 9 & 0.522 & 0.610 & 0.571 & 0.603 & 0.606 & 0.562 & 0.582 & 0.571 \\
10 & 0.614 & 0.673 & 0.643 & 0.668 & 0.674 & 0.621 & 0.609 & 0.612 \\
11 & 0.692 & 0.687 & 0.694 & 0.690 & 0.697 & 0.661 & 0.621 & 0.642 \\
12 & 0.731 & 0.692 & 0.722 & 0.700 & 0.709 & 0.679 & 0.624 & 0.655 \\
13 & 0.750 & 0.694 & 0.733 & 0.704 & 0.715 & 0.686 & 0.625 & 0.661 \\
14 & 0.761 & 0.694 & 0.737 & 0.705 & 0.717 & 0.691 & 0.625 & 0.662 \\
15 & 0.772 & 0.695 & 0.739 & 0.706 & 0.718 & 0.693 & 0.625 & 0.664 \\
16 & 0.782 & 0.695 & 0.740 & 0.707 & 0.719 & 0.693 & 0.625 & 0.664 \\
17 & 0.775 & 0.695 & 0.740 & 0.707 & 0.719 & 0.694 & 0.625 & 0.664 \\
18 & 0.774 & 0.695 & 0.740 & 0.707 & 0.719 & 0.694 & 0.625 & 0.664 \\
\hline
\end{tabular}

